\title{
Erratum: Randomization in clinical studies
}

\author{
Chi-Yeon Lim ${ }^{1}$ and Junyong $\operatorname{In}^{2}$ \\ ${ }^{1}$ Department of Biostatistics, Dongguk University College of Medicine, ${ }^{2}$ Department of Anesthesiology and Pain \\ Medicine, Dongguk University Ilsan Hospital, Goyang, Korea
}

Korean J Anesthesiol 2019 June 72(3): 221-232

https://doi.org/10.4097/kja.19049

The Appendix of this article contained an error throughout in which an incorrect symbol was used for the assignment: it should be <- characters (which is the gets arrow in R), and not $\longleftarrow$ symbol in the command lines.

For example, the corrected command lines are as below:

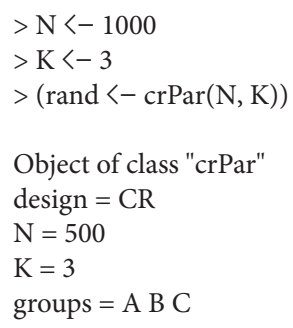

The authors would like to apologize for any inconvenience caused. 